\title{
DISCRETE HAUSDORFF TRANSFORMATIONS
}

\author{
GERALD LEIBOWITZ
}

\begin{abstract}
Let $K$ be a complex valued measurable function on $(0,1]$ such that $\int_{0}^{1} t^{-1 / n}|K(t)| d t$ is finite for some $p>1$. Let $H$ be the Hausdorff operator on $l^{\prime \prime}$ determined by the moments $\mu_{n}=$ $\int_{0}^{1} t^{n} K(t) d t$. Define $\Psi^{\prime}(z)=\int_{0}^{1} t^{z} K(t) d t$. Then for each $z$ with $\operatorname{Re} z>-1 / p, \Psi^{\prime}(z)$ is an eigenvalue of $H^{*}$. The spectrum of $H$ is the union of $\{0\}$ with the range of $\Psi$ ' on the half-plane $\operatorname{Re} z \geqq-1 / p$.
\end{abstract}

If $\mu$ is a complex Borel measure concentrated on $(0,1]$ and satisfying $\int_{0}^{1} t^{-1 / p} d|\mu|(t)<\infty$, then as shown by Hardy [2], $\mu$ determines a bounded operator $H$ on $l^{p}$, as follows: $(H a)(n)=\sum_{k=0}^{n}\left(\begin{array}{l}n \\ k\end{array}\right) \mu_{k, n-k} a(k)(n=0,1,2, \cdots)$ where $\mu_{k, j}=\int_{0}^{1}(1-t)^{j} t^{k} d \mu(t)$. Furthermore, $\|H\|_{p} \leqq \int_{0}^{1} t^{-1 / p} d|\mu|(t)$. In [4], Rhoades studied the operators $H$ which correspond to totally regular Hausdorff summability methods; here the measures $\mu$ are probability measures. He showed that in this case the norm of $H$ on $l^{p}$ is exactly $\int_{0}^{1} t^{-1 / p} d \mu(t)$, that $H$ has no eigenvectors in $l^{p}$, and that $\mu_{k}=\mu_{k, 0}$ is an eigenvalue of the adjoint $H^{*}$ (which acts on $l^{q}, 1 / p+1 / q=1$ ). Although the $\mu_{k}$ depend on $\mu$, the associated eigenvectors do not; indeed, $H^{*} b_{k}=$ $\mu_{k} b_{k}$ where $b_{k}=\Delta^{k} e_{0}$ and $e_{0}$ is the unit vector $\{1,0,0,0, \cdots\}$ in $l^{q}$. Using this observation we are able to extend the results of Rhoades concerning the spectra of the Gamma methods $\Gamma_{a}^{1}(a>1 / p)$ to the general class of operators $H$ corresponding to absolutely continuous measures $\mu$. Our theorem expresses the spectrum as the compactified range of the Mellin transform of $t^{1 / q} d \mu(t)$ over the half-plane $\operatorname{Im} s \geqq 0$.

Throughout, $p$ is a real number greater than one.

It is shown in [3] that for each complex number $\lambda$ such that $\operatorname{Re} \lambda^{-1}>1 / q$, the sequence $g_{\lambda}$ defined by the generating function

$$
(1-w)^{1 / \lambda-1}=\sum_{n=0}^{\infty} g_{\lambda}(n) w^{n}
$$

belongs to $l^{\prime \prime}$ (and is an eigenvector of the Cesàro operator). This is

Received by the editors June 2, 1972 and, in revised form, August 31, 1972.

AMS (MOS) subject classifications (1970). Primary 47A10, 40 G05.

(c) American Mathematical Society 1973 
equivalent to the following: if $\operatorname{Re} z>-1 / p$, then the sequence $f_{z}$ given by $(1-w)^{z}=\sum_{n=0}^{\infty} f_{z}(n) w^{n}$ is an element of $l^{q}$.

THEOREM 1. Let $\Psi(z)=\int_{0}^{1} t^{z} d \mu(t)$. Then $\Psi$ is continuous on the halfplane $\operatorname{Re} z \geqq-1 / p$ and analytic on $\operatorname{Re} z>-1 / p$. Moreover, for each $z$ the open half-plane, $f_{z}$ is an eigenvector of $H^{*}$ corresponding to the eigenvalue $\Psi(z)$.

Proof. We have

$$
\begin{aligned}
\Psi(z)(1-w)^{z} & =\int_{0}^{1}(1-(1-t+w t))^{z} d \mu(t) \\
& =\int_{0}^{1} \sum_{n=0}^{\infty} f_{z}(n)(1-t+w t)^{n} d \mu(t) .
\end{aligned}
$$

Using the binomial theorem and interchanging summation and integration (valid because of uniform convergence), we have

$$
\Psi(z)(1-w)^{z}=\sum_{n=0}^{\infty} f_{z}(n) \sum_{k=0}^{n}\left(\begin{array}{l}
n \\
k
\end{array}\right) \mu_{k, n-k} w^{k} .
$$

Since $H$ has matrix $\left(h_{r s}\right)$ where $h_{r s}=\left({ }_{s}^{r}\right) \mu_{s, r-s}$ if $s \leqq r, h_{r s}=0$ if $r<s, H^{*}$ has matrix entries $h_{n, m}^{*}=\left(\begin{array}{c}m \\ n\end{array}\right) \mu_{n, m-n}$ for $n \leqq m$ and 0 otherwise. Hence

$$
\begin{aligned}
\sum_{n=0}^{\infty}\left(H^{*} f_{z}\right)(n) w^{n} & =\sum_{n=0}^{\infty}\left(\sum_{m=n}^{\infty}\left(\begin{array}{l}
m \\
n
\end{array}\right) \mu_{n, m-n} f_{z}(m)\right) w^{n} \\
& =\sum_{m=0}^{\infty}\left(\sum_{n=0}^{m}\left(\begin{array}{l}
m \\
n
\end{array}\right) \mu_{n, m-n} w^{n}\right) f_{z}(m) .
\end{aligned}
$$

We conclude that $H^{*} f_{z}=\Psi^{*}(z) f_{z}$.

THEOREM 2. Suppose that $d \mu(t)=K(t) d t$ where $K$ is measurable and $\int_{0}^{1} t^{-1 / p}|K(t)| d t<\infty$. Then the spectrum of the Hausdorff transformation $H$ defined by $\mu$ on $l^{p}$ is precisely the closure of the set of values assumed by $\Psi(z)=\int_{0}^{1} t^{z} d \mu(t)$ on the half-plane $\operatorname{Re} z>-1 / p$.

Proof. By Theorem 1, the spectrum of $H$ contains $\Psi(z)$ for every $z$ with $\operatorname{Re} z>-1 / p$. Thus it will suffice, in order to prove the theorem, to show that for every $\lambda \neq 0$ which is not in the range of $\Psi$ on $\operatorname{Re} z \geqq-1 / p$, the sequence $1 /\left(\lambda-\mu_{n}\right)$ is the moment sequence of a complex measure $v$ satisfying the integrability condition $\int_{0}^{1} t^{-1 / p} d|v|(t)<\infty$.

But this is a direct consequence of the Gelfand theory for commutative 
Banach algebras. Let us define a function $h$ on the positive axis by setting $h(x)=e^{-x / q} K\left(e^{-x}\right), 0 \leqq x<\infty$. Then $h \in L^{1}(0, \infty)$ and by hypothesis, the complex Fourier transform

$$
\hat{h}(\xi)=\int_{0}^{\infty} e^{i \xi x} h(x) d x
$$

does not assume the value $\lambda$ anywhere in the half-plane $\Pi=\{\xi: \operatorname{Im} \xi \geqq 0\}$. Since the complex homomorphisms of the Banach algebra $L^{1}(0, \infty)$ are precisely the maps $h \rightarrow \hat{h}(\xi), \xi \in \Pi$, it follows from the Gelfand theory that there exists some $h_{0} \in L^{1}(0, \infty)$ such that $\lambda^{-1} h+\lambda h_{0}=h * h_{0}$, where * represents convolution. (See [1].) In particular, $(\lambda-\hat{h}(\xi))^{-1}=\lambda^{-1}-\hat{h}_{0}(\xi)$ for all $\xi \in \Pi$. If we set

$$
h_{0}(x)=e^{-x / q} K_{0}\left(e^{-x}\right), \quad 0 \leqq x<\infty,
$$

then the function $K_{0}$ so defined satisfies the integrability condition (indeed, $\left.\int_{0}^{1} t^{-1 / p}\left|K_{0}(t)\right| d t=\int_{0}^{\infty}\left|h_{0}(x)\right| d x\right)$, and $\left(\lambda-\mu_{n}\right)^{-1}$ is the moment sequence of the measure $v$, where $\nu=\lambda^{-1} \delta-K_{0} d t, \delta$ the unit mass at 1 . Thus the proof is complete.

Note that in view of the analyticity of $\Psi$ we can state the conclusion of Theorem 2 as follows: the spectrum of $H$ consists of 0 together with the range of $\int_{0}^{1} t^{z-1 / p} K(t) d t$ on the half-plane $\operatorname{Re} z \geqq 0$.

In the case of the Gamma methods of order one, $K(t)=a t^{a-1}$ where $a>1 / p$. The Mellin transform $\Psi(z)=a /(z+a)$, so the spectrum of $\Gamma_{a}^{1}$ on $l^{p}$ is the set of all complex $\lambda$ for which $a / \lambda=(z+1 / p)+a / c(c=$ the norm of $\left.\Gamma_{a}^{1}\right)$; i.e., those $\lambda$ such that $\operatorname{Re}(1 / \lambda) \geqq 1 / c=(a-1 / p) / a$. This is the closed disk $|\lambda-c / 2| \leqq c / 2$. So we have Rhoades' description of $\sigma\left(\Gamma_{a}^{1}\right)$.

REMARKS. 1. Our result extends to the various interpretations of the limiting case where $p=\infty$. (See [3].)

2. It follows from work of Wallen and Shields (Indiana Univ. Math. J. 20 (1971), 777-788) that each Hausdorff operator on $l^{2}$ is a bounded analytic function of the Cesàro operator and hence that when $p=2$, Theorem 2 is valid for all $\mu$, absolutely continuous or not. For $p$ in general, it is likely that Theorem 2 is valid for a wider class of measures than those considered here. (The author thanks the referee for these two observations.)

3. James Deddens has independently obtained results which are related to ours.

\section{REFERENCES}

1. R. F. Arens and I. M. Singer, Generalized analytic functions, Trans. Amer. Math. Soc. 81 (1956), 379-393. MR 17, 1226. 
2. G. H. Hardy, An inequality for Hausdorff means, J. London Math. Soc. 18 (1943), 46-50. MR 5, 65.

3. G. M. Leibowitz, Spectra of discrete Cesàro operators, Tamkang J. Math. 3 (1972).

4. B. E. Rhoades, Spectra of some Hausdorff operators, Acta Sci. Math. (Szeged) 32 (1971), 91-100.

Department of Mathematics, University of Connecticut, Storrs, Connecticut 06268 